\title{
DESEMPENHO DA CULTURA DA RÚCULA CULTIVADA EM ÉPOCA DE VERÃO EM TÚNEIS BAIXOS DE POLIETILENO PERFURADO
}

\author{
EDILAINE R. PEREIRA ${ }^{1}$, IRAN J. O. SILVA ${ }^{2}$, DANIELLA J. MOURA ${ }^{3}$, \\ SONIA M. S. PIEDADE ${ }^{4}$
}

RESUMO: O túnel baixo é um tipo de ambiente protegido utilizado, principalmente, em cultivo de espécies de pequeno porte, como alface, morango, pimentão, rúcula e pepino. Por serem estruturas de menor porte, apresentam menor custo de instalação em relação ao túnel alto. A presença de material plástico em sua cobertura proporciona um ganho térmico durante o dia, facilitando o crescimento das plantas, protegendo as culturas do impacto da chuva, dos ventos frios e das temperaturas mínimas. Esta pesquisa teve o objetivo de avaliar o efeito de filme de polietileno com diferentes intensidades de perfurações $(0 ; 5 ; 10 ; 15$ e 20\%), no microclima em túneis baixos e no desenvolvimento da cultura da rúcula (Eruca vesicaria sativa (Mill) Thell) em época de verão. Os resultados mostraram que houve diferença de produtividade entre os túneis com diferentes níveis de perfuração e houve diferenças significativas quanto às características físicas da planta em alguns tratamentos comparativos à testemunha. Quanto aos aspectos relacionados com o microclima, verificou-se, por meio de análise estatística, que a temperatura do ar observada nos horários mais críticos do dia (11 às 15 h) apresentou diferenças significativas entre o ambiente coberto com túnel sem perfuração e o solo descoberto; com relação à umidade absoluta do ar, quanto maior o nível de perfuração do plástico de cobertura do túnel, menor a umidade absoluta observada.

PALAVRAS-CHAVE: (Eruca vesicaria sativa (Mill) Thell), microclima, plasticultura.

\section{PERFORMANCE OF ROCKET PRESS CULTURE IN LOW TUNNELS WITH POLYETHYLENE PLASTIC PERFORATED IN SUMMER}

SUMMARY: Low tunnels is a type of greenhouse that is used for cultivation of species of small load, as lettuce, strawberry, sweet pepper, rocket press and cucumber. This type of greenhouse has a smaller cost of installation than other because its lower high. The plastic covering allows the gain of temperature during the day improving plant growth, protecting the crop from the impact of the rain, cold wind and low temperature. The purpose of this research was to decide the atmosphere changes with the use of polyethylene film with density fall, in low tunnel with perforated area at $0 ; 5 ; 10 ; 15$, and $20 \%$ in the plastic in the development of the rocket press culture (Eruca vesicaria sativa (Mill) Thell). The results showed different productivity between the tunnel with different holes and significative differences in physics characteristics of plants between some different treatments. As for the inside atmosphere of the tunnel it could see by statistic analysis, that the air temperature in review schedule of the day had significative difference between $0 \%$ of perforation and soil without covering, and, the air absolute humidity is higher as much less is the perforated plastic covering.

KEYWORDS: (Eruca vesicaria sativa (Mill) Thell), microclimate condition, plasticulture.

\footnotetext{
${ }^{1}$ Engenheira Agrícola, Doutoranda, Escola Superior de Agricultura "Luiz de Queiroz" , ESALQ, Piracicaba - SP, Fone: (0XX19) 3429.4217 - R: 237, e-mail: erpereir@carpa.ciagri.usp.br

${ }^{2}$ Prof. Dr., Departamento de Engenharia Rural, ESALQ/USP, Piracicaba - SP, e-mail: ijosilva@ carpa.ciagri.usp.br

${ }^{3}$ Profa. Dra., Departamento de Engenharia Rural, ESALQ/USP, Piracicaba - SP, e-mail: djmoura@ carpa.ciagri.usp.br

${ }^{4}$ Profa. Dra., Departamento de Ciências Exatas, ESALQ/USP, Piracicaba - SP.

Recebido pelo Conselho Editorial em: 30-4-2001

Aprovado pelo Conselho Editorial em: 8-6-2004
}

Eng. Agríc., Jaboticabal, v.24, n.2, p.285-290, maio/ago. 2004 


\section{INTRODUÇÃO}

O uso da cobertura plástica representa um dos estágios mais avançados em tecnologia de produção vegetal, possibilitando a exploração econômica de grande parte das espécies de hortaliças (alface, morango, pepino, rúcula) fora da sua época normal de cultivo, além de conferir proteção às plantas, referentes aos prejuízos causados pelas intempéries climáticas: geada, chuva de granizo, longos períodos chuvosos e ventos frios. Segundo BURIOL et al. (1993), o princípio da técnica de utilizar perfurações no plástico de polietileno supõe que, devido ao pequeno volume de ar contido no túnel, a temperatura do ar pode ser elevada em dias de intensa radiação solar. A ventilação proporcionada pelas perfurações deve ser suficiente para evitar temperaturas diurnas prejudiciais ao cultivo.

Em estudos sobre a modificação ambiental causada por túneis baixos de polietileno, cultivados com alface a 0,$78 ; 1,57 ; 2,35$ e $3,14 \%$ de área perfurada, BURIOL et al. (1993) concluíram que a perfuração não afetou significativamente a disponibilidade de energia solar. À medida que se aumentou a densidade de perfuração, ocorreu um decréscimo no valor médio diurno na temperatura do ar, demonstrando a viabilidade de ventilar o túnel baixo através da perfuração do plástico. Fatos como esses também foram concluídos por MONTEIRO et al. (2001), em trabalho com plástico perfurado a $0 ; 5 ; 10 ; 15$ e $20 \%$ de perfuração.

STRECK et al. (1994), determinando o efeito do filme de polietileno com 0,$78 ; 1,57 ; 2,35$ e 3,14\% de perfuração em túneis baixos, sobre o crescimento da alface em Santa Maria - RS, concluíram que o crescimento da cultura é favorecido no ambiente sob os túneis em relação ao ambiente externo, uma vez que se obtiveram maior número de folhas, área foliar e massa seca nos cultivos sob túnel.

Trabalhando com o cultivo da alface em túneis baixos sob diferentes níveis de perfuração, MONTEIRO et al. (2001) concluíram haver valores mais elevados de produção de massa verde, massa seca e altura das plantas no interior dos túneis, comparado ao tratamento a céu aberto, porém não foram verificadas diferenças significativas nas produções de massa seca e massa verde entre o tratamento com plástico não perfurado e os tratamentos com perfurações.

Esta pesquisa teve o objetivo de avaliar o efeito de filmes de polietileno com diferentes densidades de perfuração $(0 ; 5 ; 10 ; 15$ e $20 \%)$, no microclima em túneis baixos cobertos e no desenvolvimento da cultura da rúcula (Eruca vesicaria sativa (Mill) Thell.) em época de verão.

\section{MATERIAL E MÉTODOS}

O experimento foi conduzido no período de 30-1-2001 a 6-3-2001, na área experimental do Núcleo de Pesquisa em Ambiência (NUPEA), junto ao Departamento de Engenharia Rural da Escola Superior de Agricultura "Luiz de Queiroz" da Universidade de São Paulo (ESALQ-USP), no

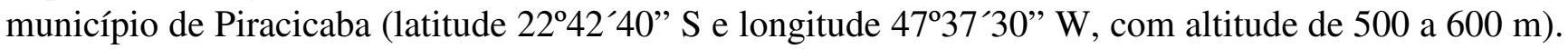
O clima da região, segundo a classificação de Köeppen, é Cwa, relativamente seco no inverno e chuvoso no verão.

Neste experimento, utilizaram-se seis canteiros, sendo cinco deles cobertos com polietileno transparente de baixa densidade (PEBD) em forma de túneis baixos semicirculares e um sem nenhum tipo de cobertura, como testemunha. Os canteiros foram construídos nas dimensões de $1,2 \mathrm{~m}$ de largura e 6,0 $\mathrm{m}$ de comprimento, separados entre si de $2 \mathrm{~m}$. Foram estudados seis diferentes tratamentos: PEBD sem perfuração, 5; 10; 15 e 20\% de perfuração, além do solo descoberto.

Foi implantada nessa área a cultura da rúcula (Eruca vesicaria sativa (Mill) Thell.) em época de verão. A cultura foi semeada diretamente em sulcos, $\operatorname{com} 0,5 \mathrm{~cm}$ de profundidade e espaçados entre si 
em $30 \mathrm{~cm}$. A cultura foi irrigada com o sistema de irrigação por gotejamento e foram aplicados turnos de rega diários de acordo com as necessidades da planta e obedecendo-se às recomendações de aplicação de $3 \mathrm{~L} \mathrm{~m}^{-2}$ (MINAME \& TESSARIOLI NETO, 1998).

Foram utilizados em cada tratamento termoigrômetros de leitura digital que forneceram dados de temperatura média e umidade relativa média do ar. Tais equipamentos foram abrigados na parte central dos canteiros, sob um anteparo de madeira que serviu de apoio e abrigo da insolação direta de radiação solar. No período de 15 dias após a semeadura, os túneis foram cobertos com o plástico de polietileno transparente e após 20 dias fez-se a colheita da rúcula.

Os dados climáticos foram coletados durante o verão, no período das 8 às $18 \mathrm{~h}$, com freqüência de hora em hora, e as variáveis-resposta do experimento foram utilizadas para comparação dos tratamentos.

Para processar o estudo da massa seca, foram colhidas, ao acaso, dez plantas por canteiro. Segundo metodologia adotada por MONTEIRO et al. (2001), após pesadas, as plantas foram mantidas em estufa de ventilação forçada até atingir massa constante.

A fim de analisar as características da planta, foram amostradas mais dez plantas por parcela e retirados valores quanto ao número de folhas, largura, altura e peso das folhas. Plantas numa área de $2 \mathrm{~m}^{2}$ por canteiro foram coletadas e pesadas imediatamente após, a fim de obter a produtividade por área em cada tratamento $\left(\mathrm{g} \mathrm{m}^{-2}\right)$.

O delineamento estatístico adotado foi o de blocos ao acaso, com os dados sendo estudados por meio do Teste de Tukey, a $1 \%$ de probabilidade da análise de variância e comparados entre si pelo pacote estatístico Statistics Analisys System (SAS).

Os diferentes níveis de perfuração foram considerados como tratamentos, e os horários de coleta de dados como blocos, sendo efetuadas cinco repetições.

\section{RESULTADOS E DISCUSSÃO}

Por meio da análise estatística realizada para a variável temperatura média do ar e analisando-se os dados obtidos no período de verão (Tabela 1), pode-se afirmar que, nos horários mais críticos do dia (11 às $15 \mathrm{~h}$ ), houve diferença significativa entre o tratamento sem túnel e os demais tratamentos com plástico perfurado. Todas as densidades de perfuração estudadas proporcionaram valores significativamente superiores ao ambiente externo, variando de 4,6 a $7,6^{\circ} \mathrm{C}$ superior a esse. O túnel com $0 \%$ de perfuração chegou a atingir, em média, $13,4^{\circ} \mathrm{C}$ superior à testemunha, comprovando que as perfurações foram suficientes para proporcionar ambiente mais bem ventilado, diminuindo a temperatura média do ar.

Quanto aos resultados da análise de umidade absoluta do ar no interior dos túneis baixos (Tabela 2), os tratamentos apresentaram valores em média, nos horários mais críticos do dia, em ordem decrescente para $0 ; 5 ; 10 ; 15$ e $20 \%$ de perfuração. Quanto menor a área perfurada, maior a resistência ao fluxo turbulento de vapor d'água e maior a dificuldade de trocas com o meio externo, o que contribui para maior retenção de vapor no interior dos túneis e aumento da umidade do ar.

Outro fator que pode ter influenciado na quantidade de vapor d'água foi uma certa diferenciação do controle estomático entre os tratamentos devido aos diferentes níveis de perfuração da cobertura plástica do túnel baixo, provocando diferentes taxas de evapotranspiração e, conseqüentemente, diferente umidade absoluta. Com maior perfuração, há maior circulação do ar no interior dos túneis, havendo variação no déficit de saturação do ar e possível alteração no balanço de radiação no que diz respeito às ondas longas. 
TABELA 1. Média da temperatura do ar durante a coleta de dados de verão para o cultivo da rúcula (Eruca vesicaria sativus (Mill) Thell).

\begin{tabular}{cccccc}
\hline \multirow{2}{*}{ Tratamento* $^{*}$} & \multicolumn{5}{c}{ Temperatura Média do Ar $\left({ }^{\circ} \mathrm{C}\right)^{*}$} \\
\cline { 2 - 5 } & 11 horas & 12 horas & 13 horas & 14 horas & 15 horas \\
\hline Testemunha & $32,5 \mathrm{c}$ & $34,2 \mathrm{c}$ & $35,3 \mathrm{c}$ & $35,6 \mathrm{~d}$ & $35,3 \mathrm{c}$ \\
$0 \%$ & $45,7 \mathrm{a}$ & $47,5 \mathrm{a}$ & $51,5 \mathrm{a}$ & $47,6 \mathrm{a}$ & $47,4 \mathrm{a}$ \\
$5 \%$ & $39,1 \mathrm{~b}$ & $41,2 \mathrm{~b}$ & $42,8 \mathrm{~b}$ & $42,8 \mathrm{bc}$ & $41,6 \mathrm{~b}$ \\
$10 \%$ & $38,5 \mathrm{~b}$ & $41,0 \mathrm{~b}$ & $44,9 \mathrm{~b}$ & $42,5 \mathrm{bc}$ & $43,6 \mathrm{ab}$ \\
$15 \%$ & $37,2 \mathrm{~b}$ & $39,4 \mathrm{~b}$ & $40,7 \mathrm{~b}$ & $39,8 \mathrm{~b}$ & $38,7 \mathrm{bc}$ \\
$20 \%$ & $38,7 \mathrm{~b}$ & $41,3 \mathrm{~b}$ & $44,4 \mathrm{~b}$ & $43,4 \mathrm{~b}$ & $43,2 \mathrm{ab}$ \\
\hline
\end{tabular}

* médias seguidas pela mesma letra nas colunas não diferem estatisticamente entre si, pelo teste de Tukey, a $1 \%$ de probabilidade.

TABELA 2. Média da umidade absoluta do ar durante a coleta de dados de verão para o cultivo da rúcula (Eruca vesicaria sativus (Mill) Thell).

\begin{tabular}{ccccccc}
\hline \multirow{2}{*}{ Tratamento } & \multicolumn{5}{c}{ Umidade Absoluta do Ar $\left(\mathrm{g}\right.$ vapor $\mathrm{m}^{-3}$ de ar)* } \\
\cline { 2 - 7 } & 10 horas & 11 horas & 12 horas & 13 horas & 14 horas & 15 horas \\
\hline Testemunha & $19,65 \mathrm{~d}$ & $19,56 \mathrm{~d}$ & $18,75 \mathrm{c}$ & $18,27 \mathrm{c}$ & $17,82 \mathrm{c}$ & $17,79 \mathrm{c}$ \\
$0 \%$ & $42,36 \mathrm{a}$ & $47,30 \mathrm{a}$ & $48,01 \mathrm{a}$ & $54,76 \mathrm{a}$ & $47,19 \mathrm{a}$ & $48,32 \mathrm{a}$ \\
$5 \%$ & $31,11 \mathrm{~b}$ & $32,85 \mathrm{~b}$ & $34,55 \mathrm{~b}$ & $33,64 \mathrm{~b}$ & $30,38 \mathrm{~b}$ & $30,51 \mathrm{~b}$ \\
$10 \%$ & $27,76 \mathrm{bc}$ & $28,91 \mathrm{bc}$ & $31,09 \mathrm{~b}$ & $34,77 \mathrm{~b}$ & $31,02 \mathrm{~b}$ & $29,98 \mathrm{~b}$ \\
$15 \%$ & $27,47 \mathrm{bc}$ & $28,72 \mathrm{bc}$ & $29,23 \mathrm{~b}$ & $31,79 \mathrm{~b}$ & $28,82 \mathrm{~b}$ & $25,60 \mathrm{~b}$ \\
$20 \%$ & $25,84 \mathrm{c}$ & $27,24 \mathrm{c}$ & $27,66 \mathrm{~b}$ & $27,59 \mathrm{~b}$ & $25,16 \mathrm{~b}$ & $24,09 \mathrm{bc}$ \\
\hline
\end{tabular}

* médias seguidas pela mesma letra nas colunas, não diferem estatisticamente entre si, pelo teste de Tukey, a $1 \%$ de probabilidade.

Analisando-se as características da planta com relação ao número de folhas produzidas, pode-se concluir que não houve diferença significativa entre o tratamento com túnel baixo, coberto com plástico sem perfuração e os demais tratamentos, inclusive o tratamento-testemunha (Tabela 3).

$\mathrm{Na}$ análise do comprimento, o tratamento em túnel sem perfuração na cobertura plástica foi o que apresentou diferença significativa com relação aos demais tratamentos, demonstrando valores médios inferiores em até $6,5 \mathrm{~cm}$, quando comparado ao ambiente externo (Tabela 3).

Recomenda-se um manuseio diferenciado nos tratamentos que utilizem túnel baixo com cobertura plástica sem perfuração nos períodos de verão em que deve ser feito o levantamento das laterais nos horários críticos do dia (período mais quente), promovendo, dessa forma, maior circulação de ar no interior do ambiente, evitando aquecimento excessivo no ambiente, a ponto de provocar a morte das plantas.

Quanto à produtividade, pode-se notar pela Figura 1 a superioridade da produção de rúcula em túneis baixos com $15 \%$ de área perfurada. Durante o verão, o túnel com $15 \%$ de área perfurada apresentou produção 57\% superior à testemunha, comprovando resultados obtidos por MONTEIRO et al. (2000), que encontraram valores superiores de produção de alface em túnel baixo com 15\% de área perfurada, durante a primavera.

O túnel coberto com plástico sem perfuração produziu número de plantas inferior à testemunha, acarretando baixa produtividade. $\mathrm{O}$ ambiente coberto com plástico sem perfuração apresentou elevada temperatura e umidade do ar e maior camada de gotículas de água sob o plástico, afetando a 
interceptação da radiação solar incidente e influenciando diretamente o crescimento e o desenvolvimento da cultura.

TABELA 3. Características da folha da rúcula (Eruca vesicaria sativus (Mill) Thell) durante a coleta de dados de verão.

\begin{tabular}{ccccc}
\hline \multirow{2}{*}{ Tratamentos* } & \multirow{2}{*}{ Número de Folhas* } & \multicolumn{3}{c}{ Características da Folha } \\
\cline { 3 - 5 } & & Comprimento $(\mathrm{cm})^{*}$ & Largura $(\mathrm{cm}) *$ & Massa $(\mathrm{g}) *$ \\
\hline Testemunha & $6,6 \mathrm{ab}$ & $24,5 \mathrm{a}$ & $6,9 \mathrm{a}$ & $3,4 \mathrm{ab}$ \\
$0 \%$ & $5,3 \mathrm{~b}$ & $18,0 \mathrm{~b}$ & $4,2 \mathrm{c}$ & $1,3 \mathrm{c}$ \\
$5 \%$ & $7,5 \mathrm{ab}$ & $26,8 \mathrm{a}$ & $6,8 \mathrm{ab}$ & $3,6 \mathrm{ab}$ \\
$10 \%$ & $6,7 \mathrm{ab}$ & $25,5 \mathrm{a}$ & $5,6 \mathrm{~b}$ & $3,7 \mathrm{ab}$ \\
$15 \%$ & $7,1 \mathrm{ab}$ & $27,0 \mathrm{a}$ & $6,7 \mathrm{~b}$ & $4,1 \mathrm{a}$ \\
$20 \%$ & $8,4 \mathrm{a}$ & $25,8 \mathrm{a}$ & $5,7 \mathrm{~b}$ & $2,7 \mathrm{~b}$ \\
\hline
\end{tabular}

* médias seguidas pela mesma letra nas colunas não diferem estatisticamente entre si, pelo teste de Tukey, a $1 \%$ de probabilidade.

A ocorrência de elevadas temperaturas ocorridas durante essa época do ano, com máximas que atingiram $48^{\circ} \mathrm{C}$ no interior dos túneis baixos sem perfuração, prejudicou a produção de rúcula. $\mathrm{O}$ manejo com abertura das laterais no tratamento com túnel baixo sem perfuração na cobertura plástica é o mais recomendado nessa época, por proporcionar maiores chances de melhor desenvolvimento à cultura.

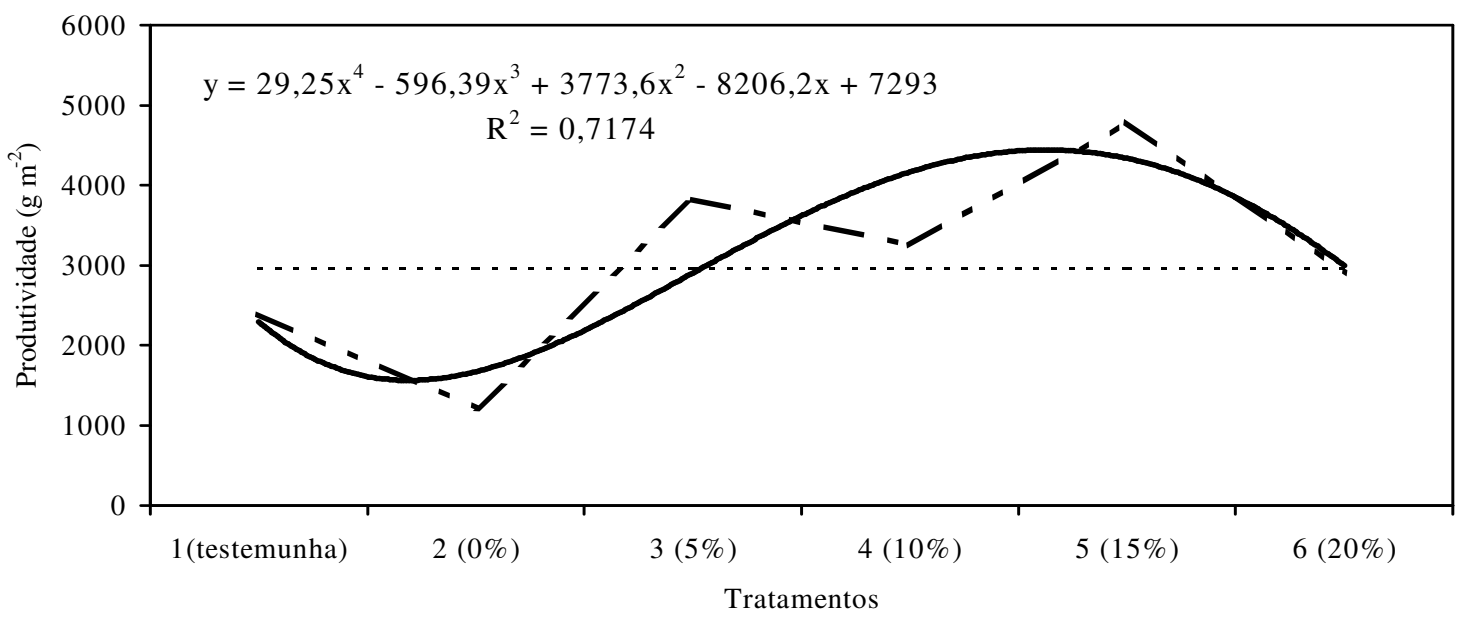

... - Produtividade testemunha - Produtividade verao $\longrightarrow$ Polinômio (Produtividade verao)

FIGURA 1. Representação do R quadrático na produtividade da rúcula (Eruca vesicaria sativus (Mill) Thell) durante coleta de dados de verão.

\section{CONCLUSÕES}

A temperatura média do ar no interior dos túneis baixos apresentou diferença significativa comparado ao tratamento a céu aberto. O tratamento com $20 \%$ de perfuração apresentou maior diferença de umidade absoluta comparado ao tratamento sem a cobertura dos túneis baixos. Devido à produtividade inferior ocorrida sob túnel baixo sem perfuração da cobertura plástica de polietileno, recomenda-se o manejo durante o dia, com abertura das laterais na época do verão. A implantação do ambiente protegido sob túneis baixos para o cultivo da rúcula em época de verão torna-se uma alternativa bastante interessante. 


\section{REFERÊNCIAS BIBLIOGRÁFICAS}

BURIOL, G.A.; SCHNEIDER, F.M.; STRECK, N.A.; MEDEIROS, S.L.P. Modificação ambiental causada por túneis baixos de polietileno transparente perfurado cultivados com alface. Ciência Rural, Santa Maria, v.23, n.3, p.261-6, 1993.

MINAMI, K.; TESSARIOLI NETO, J. A cultura da rúcula. Piracicaba: ESALQ, 1998. 19 p. (Série Produtor Rural)

MONTEIRO, J.E.B.; SILVA, I.J.O.; PIEDADE, S.M.S. Uso de filme plástico perfurado em túneis baixos (II) cultivados com alface (Lactuca sativa $\mathrm{L}$.). In: CONGRESSO BRASILEIRO DE AGROMETEOROLOGIA, 12., 2001, Fortaleza. Anais... Jaboticabal: Sociedade Brasileira de Agrometeorologia, 2001. p.777-8.

STRECK, N.A.; BURIOL, G.A.; ANDRIOLO, J.L. Crescimento da alface em túneis baixos com filme de polietileno perfurado. Ciência Rural, Santa Maria, v.24, n.2, p.235-40, 1994. 